Bull. Korean Math. Soc. 42 (2005), No. 3, pp. 443-451

\title{
GRISLY DEDUCTIVE SYSTEMS OF BL-ALGEBRAS
}

\author{
Young Bae Jun And Jung Mi Ko
}

\begin{abstract}
The notion of grisly deductive systems of a BL-algebra is introduced. Conditions for a deductive system to be a grisly deductive system are given. Extension property for a grisly deductive system is established
\end{abstract}

\section{Introduction}

Various problems in system identification involve characteristic which are essentially non-probabilistic in nature [9]. In response to this situation L. A. Zadeh introduced in 1965 fuzzy set theory as an alternative to probability theory. His fundamental idea consists in understanding lattice-valued maps as generalized characteristic functions of some new kind of subsets, so-called fuzzy sets, of a given universe. For historical reasons we quote Zadeh's original definition (cf. [10]). Fuzzy logic grows as a new discipline from the necessity to deal with vague data and imprecise information caused by the indistinguishability of objects in certain experimental environments. As mathematical tools fuzzy logic is only using $[0,1]$-valued maps and certain binary operations $*$ on the real unit interval $[0,1]$ known also as left-continuous $t$-norms. It took sometime to understand partially ordered monoids of the form $([0,1], \leq, *)$ as algebras for $[0,1]$-valued interpretations of a certain type of non-classical logicthe so-called monoidal logic. BL-algebras arise naturally in the analysis of the proof theory of propositional fuzzy logics. Indeed, Basic fuzzy logic, BL for short, and its corresponding BL-algebras, were introduced by Hájek (see [3] and the references given there) with the purpose of formalizing the many-valued semantics induced by the continuous $t$-norms on the real unit interval $[0,1]$. As a first step, Hájek showed that a propositional formula is provable in BL if and only if it is a tautology in any linearly ordered BL-algebra. In [5], Ko and Kim investigated

Received September 30, 2003.

2000 Mathematics Subject Classification: 03B52, 94D05, 06D35.

Key words and phrases: (implicative) BL-algebra, (grisly) deductive system. 
some properties of BL-algebras, and they [6] also studied relationships between closure operators and BL-algebras. Jun and Ko gave characterizations of a deductive system and discussed how to generate a deductive system by a set (cf. [4]). Turunen[7] studied Boolean deductive systems and implicative deductive systems. He proved that a BL-algebra $L$ has a proper Boolean deductive system if and only if $L$ is bipartite.

In this paper, we introduce the notion of grisly deductive systems of a BL-algebra. We give conditions for a deductive system to be a grisly deductive system, and establish an extension property for a grisly deductive system. BL-algebras, MV-algebras, and lattice implication algebras are closely related. Thus, all results in this paper will contribute much to studying MV-algebras and lattice implication algebras.

\section{Preliminaries}

A lattice $(L ; \leq, \wedge, \vee, \odot, \rightsquigarrow, 0,1)$ is called a residuated lattice if it satisfies the following conditions:

(R1) $(L ; \odot, 1)$ is a commutative monoid,

(R2) $(\forall x, y, z \in L)(x \leq y \Rightarrow x \odot z \leq y \odot z)$,

(R3) $(\forall x, y, z \in L)(x \odot y \leq z \Leftrightarrow x \leq y \leadsto z)$.

Definition 2.1. [3] A $B L$-algebra is a residuated lattice $(L ; \leq, \wedge, \vee$, $\odot, \rightsquigarrow, 0,1)$ that satisfies the following conditions:

(B1) $(\forall x, y \in L)(x \wedge y=x \odot(x \leadsto y))$,

(B2) $(\forall x, y \in L)(x \vee y=((x \rightsquigarrow y) \rightsquigarrow y) \wedge((y \leadsto x) \rightsquigarrow x))$,

(B3) $(\forall x, y \in L)((x \rightsquigarrow y) \vee(y \rightsquigarrow x)=1)$.

EXAMPLE 2.2. [5] Let $X$ be a nonempty set and let $\mathcal{P}(X)$ be the family of all subsets of $X$. Define operations $\odot$ and $\rightsquigarrow$ by

$$
A \odot B=A \cap B \text { and } A \rightsquigarrow B=A^{c} \cup B
$$

for all $A, B \in \mathcal{P}(X)$, respectively. Then $(\mathcal{P}(X), \subset, \cap, \cup, \odot, \rightsquigarrow, \emptyset, X)$ is a BL-algebra.

We call $\mathcal{P}(X)$ the power $B L$-algebra of $X$.

Proposition 2.3. $[3,8]$ In a $B L$-algebra $(L ; \leq, \wedge, \vee, \odot, \rightsquigarrow, 0,1)$, we have the following properties:

(p1) $(\forall x \in L)(x=1 \leadsto x)$,

(p2) $(\forall x \in L)(1=x \rightsquigarrow x)$,

(p3) $(\forall x, y \in L)(x \odot y \leq x, y)$,

(p4) $(\forall x, y \in L)(x \odot y \leq x \wedge y)$, 
(p5) $(\forall x, y \in L)(y \leq x \rightsquigarrow y)$,

(p6) $(\forall x, y \in L)(x \odot y \leq x \rightsquigarrow y)$,

(p7) $(\forall x, y \in L)(x \leq y \Leftrightarrow 1=x \rightsquigarrow y)$,

(p8) $(\forall x, y \in L)(x=y \Leftrightarrow 1=x \rightsquigarrow y=y \rightsquigarrow x)$,

(p9) $(\forall x, y \in L)(x \odot(x \rightsquigarrow y) \leq y)$,

(p10) $(\forall x, y, z \in L)(x \rightsquigarrow(y \rightsquigarrow z)=y \rightsquigarrow(x \rightsquigarrow z))$,

(p11) $(\forall x, y, z \in L)((x \rightsquigarrow y) \rightsquigarrow(x \rightsquigarrow z) \leq x \rightsquigarrow(y \rightsquigarrow z))$,

(p12) $(\forall x, y, z \in L)(x \rightsquigarrow y \leq(z \rightsquigarrow x) \rightsquigarrow(z \rightsquigarrow y))$,

(p13) $(\forall x, y, z \in L)(x \leq y \Rightarrow z \rightsquigarrow x \leq z \rightsquigarrow y, y \rightsquigarrow z \leq x \rightsquigarrow z)$.

A BL-algebra $L$ is said to be implicative [4] if it satisfies the following inequality:

$$
(\forall x, y, z \in L)(x \rightsquigarrow(y \rightsquigarrow z) \leq(x \rightsquigarrow y) \rightsquigarrow(x \rightsquigarrow z)) .
$$

Recall that the power BL-algebra $\mathcal{P}(X)$ of a set $X$ is an implicative BL-algebra (see [4]).

\section{Grisly deductive systems}

In what follows, let $L$ denote a BL-algebra unless otherwise specified. For every $a_{1}, a_{2}, \cdots, a_{n} \in L$, we define

$$
P\left(a_{1}, a_{2}, \cdots, a_{n-1} \backslash a_{n}\right):= \begin{cases}a_{n} & \text { if } n=1, \\ a_{1} \leadsto P\left(a_{2}, a_{3}, \cdots, a_{n-1} \backslash a_{n}\right) & \text { if } n>1 .\end{cases}
$$

Definition 3.1. $[2,7,8]$ A subset $D$ of $L$ is called a deductive system of $L$ if it satisfies the following conditions:

(ds1) $1 \in D$,

(ds2) $(\forall x, y \in L)(x \in D, P(x \backslash y) \in D \Rightarrow y \in D)$.

Proposition 3.2. [5] Let $D$ be a nonempty subset of $L$. Then $D$ is a deductive system of $L$ if and only if it satisfies:

(ds3) $(\forall a, b \in D)(a \odot b \in D)$,

(ds4) $(\forall a \in D)(\forall b \in L)(a \leq b \Rightarrow b \in D)$.

Definition 3.3. Let $D$ be a nonempty subset of $L$. Then $D$ is called a grisly deductive system of $L$ if it satisfies (ds1) and (ds5) $(\forall x, y, z \in L)(P(x, y \backslash z) \in D, P(x \backslash y) \in D \Rightarrow P(x \backslash z) \in D)$.

EXAmPLE 3.4. (1) Consider the power BL-algebra $(\mathcal{P}(X), \subset, \cap, \cup$, $\odot, \rightsquigarrow, \emptyset, X)$ of a set $X$. For any $A \in \mathcal{P}(X)$, the set

$$
\mathfrak{D}:=\{B \in \mathcal{P}(X) \mid A \subset B\}
$$


is a grisly deductive system of $\mathcal{P}(X)$.

(2) Let $L=\{0, a, b, c, d, 1\}$ be a set with Hasse diagram and Cayley tables as follows:

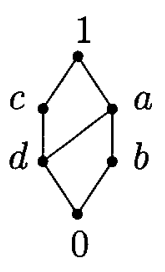

\begin{tabular}{c|cccccc}
$\odot$ & 1 & $a$ & $b$ & $c$ & $d$ & 0 \\
\hline 1 & 1 & $a$ & $b$ & $c$ & $d$ & 0 \\
$a$ & $a$ & $b$ & $b$ & $d$ & 0 & 0 \\
$b$ & $b$ & $b$ & $b$ & 0 & 0 & 0 \\
$c$ & $c$ & $d$ & 0 & $c$ & $d$ & 0 \\
$d$ & $d$ & 0 & 0 & $d$ & 0 & 0 \\
0 & 0 & 0 & 0 & 0 & 0 & 0
\end{tabular}

\begin{tabular}{c|cccccc}
$\rightsquigarrow$ & 1 & $a$ & $b$ & $c$ & $d$ & 0 \\
\hline 1 & 1 & $a$ & $b$ & $c$ & $d$ & 0 \\
$a$ & 1 & 1 & $a$ & $c$ & $c$ & $d$ \\
$b$ & 1 & 1 & 1 & $c$ & $c$ & $c$ \\
$c$ & 1 & $a$ & $b$ & 1 & $a$ & $b$ \\
$d$ & 1 & 1 & $a$ & 1 & 1 & $a$ \\
0 & 1 & 1 & 1 & 1 & 1 & 1
\end{tabular}

For every $x, y \in L$, define $x \wedge y=x \odot P(x \backslash y)$ and

$$
x \vee y=P(P(x \backslash y) \backslash y) \odot P(P(P(x \backslash y) \backslash y) \backslash P(P(y \backslash x) \backslash x)) .
$$

Then $(L ; \leq, \wedge, \vee, \odot, \rightsquigarrow, 0,1)$ is a BL-algebra. It is easily to verify that $D:=\{1, a, b\}$ is a grisly deductive system of $L$.

THEOREM 3.5. Every grisly deductive system is a deductive system.

Proof. Let $D$ be a grisly deductive system of $L$ and let $x, y \in L$ be such that $x \in D$ and $P(x \backslash y) \in D$. Then $P(1 \backslash x)=x \in D$ and $P(1, x \backslash y)=P(x \backslash y) \in D$. It follows from (p1) and (ds5) that $y=$ $P(1 \backslash y) \in D$. Hence $D$ is a deductive system of $L$.

The converse of Theorem 3.5 may not be true as seen in the following example.

EXAMPLE 3.6. Let $L$ be a BL-algebra in Example 3.4(2). Then $\{1\}$ is a deductive system of $L$, but not a grisly deductive system of $L$ since $P(d, a \backslash 0)=1 \in\{1\}$ and $P(d \backslash a)=1 \in\{1\}$, but $P(d \backslash 0)=a \notin\{1\}$.

THEOREM 3.7. If $L$ is implicative, then every deductive system is a grisly deductive system.

Proof. Let $D$ be a deductive system of an implicative BL-algebra $L$. Let $x, y, z \in L$ be such that $P(x, y \backslash z) \in D$ and $P(x \backslash y) \in D$. Since $L$ is implicative, we have

$$
P(P(x \backslash y) \backslash P(x \backslash z))=P(x, y \backslash z) \in D,
$$

and so $P(x \backslash z) \in D$ by (ds2). Hence $D$ is a grisly deductive system of $L$.

For every $w \in L$, we define a set

$$
[w):=\{x \in L \mid P(w \backslash x)=1\} .
$$


Obviously, $1, w \in[w]$. The following example shows that $[w]$ is not a deductive system of $L$ in general.

EXAMPLE 3.8. Let $L$ be a BL-algebra in Example 3.4(2). Then $[a)=$ $\{1, a\}$ is not a deductive system of $L$ since $P(a \backslash b)=a \in[a)$ and $a \in[a)$, but $b \notin[a)$.

We give a condition for $[w)$ to be a deductive system of $L$.

Theorem 3.9. Let $w$ be a fixed element of $L$. Then the set $[w]$ is a deductive system of $L$ if and only if the following implication is valid:

$$
(\forall x, y \in L)(P(w, x \backslash y)=1, P(w \backslash x)=1 \Rightarrow P(w \backslash y)=1) .
$$

Proof. Assume that $[w]$ is a deductive system of $L$ and let $x, y \in L$ be such that $P(w, x \backslash y)=1$ and $P(w \backslash x)=1$. Then $P(x \backslash y) \in[w)$ and $x \in[w)$. Since $[w]$ is a deductive system of $L$, we have $y \in[w)$, that is, $P(w \backslash y)=1$ by $(\mathrm{ds} 2)$.

Conversely, suppose that the implication (1) is valid. Let $x, y \in L$ be such that $x \in[w)$ and $P(x \backslash y) \in[w)$. Then $P(w \backslash x)=1$ and $P(w, x \backslash y)=$ 1 , which imply from hypothesis that $P(w \backslash y)=1$, i.e., $y \in[w)$. Hence $[w]$ is a deductive system of $L$.

THEOREM 3.10. If $\{1\}$ is a grisly deductive system of $L$, then the set $[w]$ is a deductive system of $L$ for all $w \in L$.

Proof. Let $x, y \in L$ be such that $x \in[w)$ and $P(x \backslash y) \in[w)$. Then $P(w \backslash x)=1 \in\{1\}$ and $P(w, x \backslash y)=1 \in\{1\}$. Since $\{1\}$ is a grisly deductive system of $L$, it follows from (ds5) that $P(w \backslash y)=1$ so that $y \in[w)$. Therefore $[w)$ is a deductive system of $L$.

For every nonempty subset $D$ of $L$ and $a \in L$, we define

$$
D_{a}:=\{x \in L \mid P(a \backslash x) \in D\} .
$$

Note that $D_{1}=D$. Let $D$ be a deductive system of $L$. Then there exists $u \in L$ such that $D_{u}$ is not a deductive system of $L$ as seen in the following example.

EXAmPLe 3.11. Let $L$ be a BL-algebra in Example 3.4(2). Consider a deductive system $D=\{1, c\}$ of $L$. Then $D_{a}=\{1, a, c, d\}$ is not a deductive system of $L$, since $P(a \backslash b)=a \in D_{a}$ and $a \in D_{a}$, but $b \notin D_{a}$.

We now provide a condition for a deductive system to be a grisly deductive system. 
Theorem 3.12. Let $D$ be a deductive system of $L$. Then $D$ is a grisly deductive system of $L$ if and only if for every $u \in L, D_{u}$ is a deductive system of $L$.

Proof. Assume that $D$ is a grisly deductive system of $L$ and let $u \in L$. Obviously, $1 \in D_{u}$. Let $x, y \in L$ be such that $P(x \backslash y) \in D_{u}$ and $x \in D_{u}$. Then $P(u, x \backslash y) \in D$ and $P(u \backslash x) \in D$. Using (ds5), we know that $P(u \backslash y) \in D$, and so $y \in D_{u}$. Hence $D_{u}$ is a deductive system of $L$.

Conversely, suppose that $D_{u}$ is a deductive system of $L$ for every $u \in L$. Let $x, y, z \in L$ be such that $P(x, y \backslash z) \in D$ and $P(x \backslash y) \in D$. Then $P(y \backslash z) \in D_{x}$ and $y \in D_{x}$, which imply from (ds2) that $z \in D_{x}$, that is, $P(x \backslash z) \in D$. Therefore $D$ is a grisly deductive system of $L$.

LEMMA 3.13. Let $D$ be a nonempty subset of $L$ such that (ds1) $1 \in D$, (ds6) $(\forall x, y, z \in L)(x \in D, P(x, y, y \backslash z) \in D \Rightarrow P(y \backslash z) \in D)$.

Then $D$ is a grisly deductive system of $L$.

Proof. Let $x, y \in L$ be such that $x \in D$ and $P(x \backslash y) \in D$. Then $P(x, 1,1 \backslash y)=P(x \backslash y) \in D$ and $x \in D$. It follows from (p1) and (ds6) that $y=P(1 \backslash y) \in D$. Hence $D$ is a deductive system of $L$. Now let $x, y, z \in L$ be such that $P(x, y \backslash z) \in D$ and $P(x \backslash y) \in D$. Using (p10) and (p12), we have

$$
P(x, y \backslash z)=P(y, x \backslash z) \leq P(P(x \backslash y) \backslash P(x, x \backslash z)),
$$

which together with (ds4) implies that $P(P(x \backslash y) \backslash P(x, x \backslash z)) \in D$. Since $P(x \backslash y) \in D$, it follows from (ds6) that $P(x \backslash z) \in D$. Therefore $D$ is a grisly deductive system of $L$.

LEMMA 3.14. Every grisly deductive system $D$ of $L$ satisfies the following implication.

(ds7) $(\forall x, y \in L)(P(x, x \backslash y) \in D \Rightarrow P(x \backslash y) \in D)$.

Proof. Straightforward.

LEMMA 3.15. Let $D$ be a grisly deductive system of $L$.

(i) If $D$ satisfies (ds7), then it also satisfies (ds8) $(\forall x, y, z \in L)(P(x, y \backslash z) \in D \Rightarrow P(P(x \backslash y) \backslash P(x \backslash z)) \in D)$.

(ii) If $D$ satisfies (ds8), then it also satisfies (ds6).

Proof. (i) Let $x, y, z \in L$ be such that $P(x, y \backslash z) \in D$. Note that

$$
P(x, y \backslash z) \leq P(x, P(x \backslash y), x \backslash z)=P(x, x \backslash P(P(x \backslash y) \backslash z))
$$


which implies from (ds4) that $P(x, x \backslash P(P(x \backslash y) \backslash z)) \in D$. Using (ds7) and $(\mathrm{p} 10)$, we have

$$
P(P(x \backslash y) \backslash P(x \backslash z))=P(x \backslash P(P(x \backslash y) \backslash z)) \in D .
$$

(ii) Let $x, y, z \in L$ be such that $x \in D$ and $P(x, y, y \backslash z) \in D$. Then $P(y, y \backslash P(x \backslash z))=P(y, y, x \backslash z) \in D$ by (p10). It follows from (p1), (p2), (p10), and (ds8) that

$$
P(x \backslash P(y \backslash z))=P(y \backslash P(x \backslash z))=P(P(y \backslash y) \backslash P(y \backslash P(x \backslash z))) \in D .
$$

Since $D$ is a deductive system of $L$ and $x \in D$, we have $P(y \backslash z) \in D$ by (ds2). This completes the proof.

Combining Theorem 3.5 and Lemmas 3.13, 3.14 and 3.15, we have

Theorem 3.16. Let $D$ be a nonempty subset of $L$. Then the following are equivalent.

(i) $D$ is a grisly deductive system of $L$.

(ii) $D$ is a deductive system of $L$ that satisfies the condition (ds7).

(iii) $D$ is a deductive system of $L$ that satisfies the condition (ds8).

(iv) $D$ satisfies conditions (ds1) and (ds6).

THEOREM 3.17 (Extension property for grisly deductive systems). Let $C$ and $D$ be deductive systems of $L$ such that $C \subset D$. If $C$ is a grisly deductive system of $L$, then so is $D$.

Proof. Let $x, y, z \in L$ be such that $P(x, y \backslash z) \in D$. Then

$$
P(x, y \backslash P(P(x, y \backslash z) \backslash z))=P(P(x, y \backslash z) \backslash P(x, y \backslash z))=1 \in C .
$$

It follows from (ds8) that $P(P(x \backslash y) \backslash P(x \backslash P(P(x, y \backslash z) \backslash z))) \in C$ so that

$$
\begin{aligned}
& P(P(x, y \backslash z) \backslash P(P(x \backslash y), x \backslash z)) \\
= & P(P(x \backslash y) \backslash P(P(x, y \backslash z) \backslash P(x \backslash z))) \\
= & P(P(x \backslash y) \backslash P(x \backslash P(P(x, y \backslash z) \backslash z))) \in C \subset D .
\end{aligned}
$$

Since $D$ is a deductive system of $L$ and $P(x, y \backslash z) \in D$, we have

$$
P(P(x \backslash y) \backslash P(x \backslash z))=P(P(x \backslash y), x \backslash z) \in D
$$

by (ds2). This shows that $D$ satisfies (ds8), and so $D$ is a grisly deductive system of $L$ by Theorem 3.16. 


\section{Concluding remarks}

In this paper, we introduced the notion of grisly deductive systems of a BL-algebra, and gave conditions for a deductive system to be a grisly deductive system. We established an extension property for a grisly deductive system. The results of this paper will be devoted to study of MV-algebras, lattice implication algebras, Lukasiewicz' logic, Gödel's logic and the product logic, which are different extensions of basic logic. Moreover, it will be devoted to the problem to reveal the logical content of various methods from fuzzy logic which play a specific role in fuzzy control and expert systems, e.g. Zadeh's compositional rule of inference, generalized modus ponens, min-composition, generalized quantification, etc. Some important issues for future work are: (i) developing the properties of a (grisly) deductive system, (ii) defining new deductive systems which are related to given (grisly) deductive systems, (iii) finding useful results on the new structures, and (iv) constructing the fuzzification of such structures.

\section{Acknowledgements}

The first author is an Executive Research Worker of Educational Research Institute in GSNU.

\section{References}

[1] L. Biacino and G. Gerla, An extension principle for closure operators, J. Math. Anal. Appl. 198 (1996), 1-24.

[2] A. DiNola, G. Georgescu, and L. Leustean, Boolean products of BL-algebras, J. Math. Anal. Appl. 251 (2000), 106-131.

[3] P. Hájek, Metamathematices of Fuzzy Logic, Kluwer Academic Publishers, Dordrecht, 1998.

[4] Y. B. Jun and J. M. Ko, Deductive systems of BL-algebras, Bull. Korean Math. Soc. (submitted).

[5] J. M. Ko and Y. C. Kim, Some properties of BL-algebras, J. Korea Fuzzy Logic and Intelligent Systems 11 (2001), no. 3, 286-291.

[6] Closure operators on BL-algebras, Comm. Korean Math. Soc. (submitted).

[7] E. Turunen, Boolean deductive systems of BL-algebras, Arch. Math. Logic 40 (2001), 467-473.

[8] , Mathematics behind fuzzy logic, Springer-Verlag Co., 1999

[9] L. A. Zadeh, From circuit theory to system theory, Proc. Inst. Radio Eng. 50 (1962), 856-865.

[10] , Fuzzy sets, Inform. and Control(Shenyang) 8 (1965), 338-353. 
Young Bae Jun, Department of Mathematics Education, Gyeongsang NaTIONAL UNIVERSiTy, CHINJu 660-701, KOREA

E-mail: ybjun@gsnu.ac.kr jamjana@korea.com

Jung Mi Ko, Department of Mathematics, Kangnung National University, GANGNEUNG 210-702, KOREA

E-mail: jmko@kangnung.ac.kr 